\title{
Identification and Estimation of \\ Continuous-Time, Data-Based Mechanistic (DBM) Models for Environmental Systems
}

\author{
P.C. YounG ${ }^{\dagger *}$ and H. Garnier ${ }^{\ddagger}$
}

\begin{abstract}
Initially, the paper provides an introduction to the main aspects of existing timedomain methods for identifying linear continuous-time models from discrete-time data and shows how one of these methods has been applied to the identification and estimation of a model for the transportation and dispersion of a pollutant in a river. It then introduces a widely applicable class of new, nonlinear, State-Dependent Parameter (SDP) models. Finally, the paper describes how this SDP approach has been used to identify, estimate and control a nonlinear differential equation model of global carbon cycle dynamics and global warming.
\end{abstract}

Keywords: continuous-time, stochastic, linear, instrumental variable, optimal estimation, state dependent parameter, nonlinear, environmental.

Software Availability Section:

Name of software: CAPTAIN Toolbox, Version 5.2;

developer and contact address: P. C. Young ${ }^{\dagger *}$;

year first available: 1990;

software required: Matlab ${ }^{\mathrm{TM}}$;

program language: Matlab ${ }^{\mathrm{TM}}$ language;

availability: p-coded fully functional demonstration version from the Internet at http://www.es.lancs.ac.uk/cres/captain/ 
Name of software: CONtinuous-Time System IDentification (CONTSID) Toolbox, version 4.0; developer and contact address: H. Garnier ${ }^{\ddagger}$; year first available: 1999;

software required: Matlab $^{\mathrm{TM}}$;

program language: Matlab ${ }^{\mathrm{TM}}$ language;

availability: p-coded version freely available from Internet at http://www.cran.uhp-nancy.fr/contsid/

$\dagger$ Centre for Research on Environmental Systems and Statistics, Lancaster University, Lancaster LA1 4YQ, U.K. p.young@lancaster.ac.uk

* Centre for Resource and Environmental Studies, Australian National University, Canberra, Australia.

‡ Centre de Recherche en Automatique de Nancy (CRAN - CNRS UMR 7039), Université Henri Poincaré, Nancy 1, France. hugues.garnier@cran.uhp-nancy.fr 


\section{Introduction}

In recent years, there has been increasing interest in the use of Transfer Function (TF) models in environmental science and engineering. This arises for three main reasons. First, they provide a generic approach to the data-based, stochastic modelling of linear systems; an approach that encompasses both discrete and continuous time model forms. Second, a considerable amount of research has been devoted to the statistical identification and estimation ${ }^{1}$ of TF models, so providing a rigorous statistical basis for model development. Finally, because of their heritage in the control and systems literature, TF models are in an ideal form for block diagram analysis and the interpretation of the model in serial, parallel and feedback connections of sub-systems that often have physical significance. In addition to this, more recent research has extended the domain of $\mathrm{TF}$ modelling to encompass Time Variable Parameter (TVP) and State Dependent Parameter (SDP) TF models, so allowing for their application to non-stationary and nonlinear stochastic systems.

One only has to look at recent papers in this Journal to see that many environmental models are formulated in terms of continuous-time differential equations (see e.g. Belforte et al., 2005; Zhan, 2005; Carvalho et al., 2005). As a result, the continuous-time TF model, which is simply an alternative representation of a differential equation, provides an obvious vehicle for data-based environmental modelling. Since the early 1960's a variety of different approaches have been suggested for the identification and estimation of continuous-time, linear TF models from discrete-time, sampled data (see e.g. the reviews of Young 1981; Unbehauen and Rao 1990 1998; Nielsen et al. 2000; Garnier et al. 2003). Despite this, however, such methods are not very well known or used by the environmental science and engineering communities, who tend to prefer the alternative estimation methods for discrete-time TF models (e.g. Jakeman et al. 1990; Young 2003 and the prior references therein), such as those available in the CAPTAIN Toolbox for Matlab ${ }^{\mathrm{TM}}$ and the Matlab ${ }^{\mathrm{TM}}$ System IDentification (SID) Toolbox.

However, renewed interest in continuous-time model estimation is emerging following recent tutorial sessions at the 2003 IFAC Symposium on System Identification (SYSID) in Amsterdam and the 2004 American Control Conference (ACC) in Boston, USA. In this paper, therefore, we briefly consider the available methods of estimation for continuous-time linear systems and review a method of estimation for a widely applicable class of continuous-time, nonlinear systems. In

\footnotetext{
${ }^{1}$ Here we use the statistical meanings of these words: 'identification' is the definition of the most appropriate model order and structure; and 'estimation' is the estimation of the parameters that characterize this identified model.
} 
addition, we present two examples, based on the analysis of real environmental data, that demonstrate the practical utility and efficacy of these methods. Our main aim is to demonstrate the many advantages of direct continuous-time model estimation, in relation to its discrete-time alternative, and so encourage its practical application.

The paper is presented in two main parts. The first part, in section 2., starts by reviewing various time-domain approaches to linear, continuous-time model identification and estimation, starting with the iterative Simplified Refined Instrumental Variable method for Continuous-time systems (SRIVC) method, that can be interpreted in statistically optimal and quasi-optimal terms when the transfer function model residuals are serially uncorrelated, white noise. It is also noted that this SRIVC algorithm can be extended to its more complex RIVC form, which is optimal or quasi-optimal for the case of coloured residuals. The paper then proceeds to outline the main aspects of alternative, deterministic, sub-optimal methods, before discussing the utility of the 'direct' continuous-time estimation methodology, in comparison to the alternative 'indirect' discrete-time approach (where a discrete-time model is first estimated by using standard discrete-time TF estimation methods and then converted into a continuous-time model). Finally in this first section of the paper, the practical utility of the SRIVC approach is illustrated by the its application to the 'Aggregated Dead-Zone' (ADZ) modelling of pollutant transport in a river, based on data collected during a tracer experiment.

The second part of the paper, in section 3., considers nonlinear modelling, concentrating on the estimation of State-Dependent Parameter (SDP) models, a widely applicable class of stochastic nonlinear models. The estimation methodology for SDP models follows logically from the linear methods and can describe a wide variety of nonlinear systems, including chaotic processes. Although applicable to both discrete and continuous-time systems, only the continuous-time case is considered here. The practical utility of this SDP approach is illustrated by its application to a well known set of global climate data. This first, non-parametric stage of the SDP analysis identifies the presence and nature of a temperature-dependent, negative feedback effect; and in the second, parametric stage this nonlinearity is parameterized and the resulting model is estimated from the data using a prediction error minimization approach.

Note that the paper does not attempt to review the literature on the identification and estimation of models based on Itô stochastic differential equations (see e.g. Nielsen et al. 2000; Kristensen et al. 2004, and the references on this topic therein). While this is partly through lack of space, it is also because this alternative approach has not been applied so widely and is theoretically more demanding. By contrast, the methods discussed below have been proven in many practical 
applications and are available as computationally efficient algorithms in two Matlab ${ }^{\mathrm{TM}}$ toolboxes: CAPTAIN and CONTSID.

\section{Linear Continuous-Time Model Identification}

The theoretical basis for the statistical identification and estimation of linear, continuous-time models from discrete-time, sampled data can be outlined by considering the following Single-Input, Single-Output (SISO) system ${ }^{2}$

$$
\left\{\begin{array}{l}
x(t)=\frac{B(s)}{A(s)} u(t-\tau) \\
y(t)=x(t)+e(t) .
\end{array}\right.
$$

Here $A(s)$ and $B(s)$ are the following polynomials in the derivative operator $s=d / d t$ :

$$
\begin{array}{r}
A(s)=s^{n}+a_{1} s^{n-1}+\ldots+a_{n-1} s+a_{n} \\
B(s)=b_{0} s^{m}+b_{1} s^{m-1}+\ldots+b_{m-1} s+b_{m}
\end{array}
$$

and $\tau$ is any pure time delay in time units. This Transfer Function model structure is denoted by the triad $[n, m, \tau]$. In $(1), u(t)$ is the input signal, $x(t)$ is the 'noise free' output signal and $y(t)$ is the noisy output signal. Initially, the noise $e(t)$ is considered as zero mean, white noise with Gaussian amplitude distribution, although we will see later in section 2.1 that this assumption is not restrictive. Of course, the model (1) can also be written in the following differential equation form, which is often more familiar to physical scientists

$$
\frac{d^{n} y(t)}{d t^{n}}+a_{1} \frac{d^{n-1} y(t)}{d t^{n-1}}+\ldots+a_{n} y(t)=b_{0} \frac{d^{m} u(t-\tau)}{d t^{m}}+\ldots+b_{m} u(t-\tau)+\mu(t)
$$

where $\mu(t)$ is defined as $\mu(t)=A(s) e(t)$.

Note that $s$ is used as the derivative operator in the TF model (1) because of the very close relationship to the Laplace transform operator, which is often used in this same context and allows for the incorporation of initial conditions on the variables and their derivatives. Initially, however, it is assumed that there are no major effects of any initial conditions remaining on the observed time series $y(t)$ and $u(t)$ that would complicate the estimation of the model parameters.

\footnotetext{
${ }^{2}$ Multiple-Input, Single-Output (MISO) and Multiple-Input, Multiple-Output MIMO extensions are straightforward but obviously more complex.
} 


\subsection{Optimal and Quasi-Optimal Estimation}

The more common but rather ad hoc deterministic methods for estimating continuous-time models, such as (1), from discrete-time series data are not formulated within the context of statistical optimality. Indeed, the topic of optimal statistical estimation in this continuous-time context has received only a small amount of attention (e.g. Young and Jakeman, 1980; Wang and Gawthrop, 2001; Young, 2002b). This is rather surprising because optimal statistical methods provide quantification of the uncertainty associated with the parameter estimates (see later examples in sections 2.4 aand 3.4) that can prove very useful in practice and such an approach is relatively standard in the case of discrete-time model estimation. Of course, the estimation of continuous-time models from discrete-time data is made more difficult because the input signal is not normally known over the sampling interval and has to be interpolated in some manner. However, in the case where the input is approximately constant over the sampling interval, it is possible to formulate the optimal estimation solution fairly straightforwardly. In other situations, the optimality of the algorithm will depend on the method of interpolation that is used. If this is not optimal, we refer to the algorithm as 'quasi-optimal' although such quasi-optimal methods provide results that are often very close to optimality and can provide an excellent, practical approach to continuous-time model estimation.

Following the usual Prediction Error Minimization (PEM) approach (Maximum Likelihood (ML) in the present situation because of the Gaussian assumption) for the model (1), a suitable error function $\varepsilon(t)$ is given by,

$$
\begin{aligned}
\varepsilon(t) & =y(t)-\frac{B(s)}{A(s)} u(t-\tau) \\
& =\frac{1}{A(s)}\{A(s) y(t)-B(s) u(t-\tau)\} .
\end{aligned}
$$

Minimization of a least squares criterion function in $\varepsilon(t)$, measured at the sampling instants, represents a nonlinear estimation problem and provides the basis for the response or output error estimation methods. However, since the operators commute in this linear case, the $1 / A(s)$ filter can be taken inside the brackets to yield the expression

$$
\varepsilon(t)=A(s) y_{f}(t)-B(s) u_{f}(t-\tau)
$$

or,

$$
\varepsilon(t)=s^{n} y_{f}(t)+a_{1} s^{n-1} y_{f}(t)+\ldots+a_{n} y_{f}(t)-b_{0} s^{m} u_{f}(t-\tau)-\ldots-b_{m} u_{f}(t-\tau)
$$

where the ${ }_{f}$ subscript indicates that the associated variable has been 'prefiltered' by $1 / A(s)$. The advantage of this transformation is that (8) is now linear in the unknown parameters $a_{i}, i=$ 
$1, \ldots, n ; b_{j}, j=0, \ldots, m$, so that the associated estimation model can be written in the form

$$
s^{n} y_{f}(t)=\mathbf{z}_{f}^{T}(t) \mathbf{a}+e(t)
$$

where,

$$
\begin{aligned}
\mathbf{z}_{f}(t) & =\left[-s^{n-1} y_{f}(t) \ldots-y_{f}(t) s^{m} u_{f}(t-\tau) \ldots u_{f}(t-\tau)\right]^{T} \\
\mathbf{a} & =\left[\begin{array}{llll}
a_{1} \ldots a_{n} b_{0} \ldots b_{m}
\end{array}\right]^{T} .
\end{aligned}
$$

As a result, all of the prefiltered derivatives appearing as variables in this estimation model are measurable as the inputs of the integrators that appear in the realization of the prefilter $1 / A(s)$. Thus, provided we assume that $A(s)$ is known, the estimation model (9) forms a basis for the definition of a likelihood function and ML estimation.

There are two problems with this formulation. The obvious one is, of course, that $A(s)$ is not known a priori. The less obvious one is that, in practical applications, we cannot assume that the noise $e(t)$ will have the nice white noise properties assumed above: it is likely that the noise will be a coloured noise process, say $\xi(t)$. Both of these problems can be solved by employing a similar approach to that used in the Refined Instrumental Variable (RIV) algorithm for discretetime (backward-shift operator TF) model identification and estimation (see Young and Jakeman 1979; Jakeman and Young 1979; Young 1984 and the prior references therein). Here, a 'relaxation' optimization procedure is devised that adaptively adjusts an initial estimate $\hat{A}_{0}(s)$ of $A(s)$ in an iterative algorithm until it converges on an optimal estimate of $A(s)$. The coloured noise problem is then solved conveniently by exploiting IV estimation within this iterative optimization algorithm. If the coloured noise is not modelled and estimated explicitly within the RIV algorithm, it is referred to as the Simplified Refined Instrumental Variable (SRIV) algorithm. The continuoustime version of this SRIV algorithm (SRIVC) is described fully in Young and Jakeman (1980) and outlined in Young (2002c). The SRIVC and RIV/SRIV algorithms are both available in the CAPTAIN toolbox ${ }^{3}$; and the SRIVC algorithm is also available in the CONTSID Toolbox (see software availability section). Of course, if the noise $e(t)$ is coloured or the input $u(t)$ is not constant between samples, then the above SRIVC approach to estimation is not optimal in statistical terms, although it is robust and normally yields estimates with reasonable statistical efficiency (i.e. low but not minimum variance). In the former case, it is possible to obtain quasi-optimal estimates by

\footnotetext{
${ }^{3}$ The CAPTAIN toolbox also contains optimized recursive filtering, forecasting and fixed interval smoothing algorithms for the estimation of time and state-dependent variable parameters in various models (TF, ARX, linear and harmonic regression models).
} 
modelling the coloured noise in AutoRegressive-Moving Average (ARMA) or AutoRegressive (AR) terms (see e.g. Box and Jenkins, 1970) and expanding the definition of the adaptive prefilters to account for this, as in the optimal RIV method for discrete-time systems. However, since it is well known that there are theoretical and practical problems associated with continuous-time ARMA and AR modelling, it is practically advantageous to use a hybrid approach in which the noise modelling is carried out in discrete-time terms ${ }^{4}$ (Young and Jakeman 1980; Johansson 1994; Pintelon et al. 2000). As regards the interpolation of the input signal $u(t)$, it is possible to consider optimal interpolation, for example using optimal fixed interval smoothing procedures (e.g. Young et al. 1999; Young 1999), but experience suggests that simple interpolation normally produces very good estimation results providing, of course, that the sampling interval is not too large.

Finally, it is worth noting that, if there are effects of any initial conditions on the observed time series $y(t)$ and $u(t)$ then, provided they are not too severe, they can be handled in a sub-optimal manner by the instrumental variable procedures that are an inherent and important part of the above estimation algorithms. However, the algorithms can be extended to allow for the estimation of such initial conditions, if this proves necessary because their effects are large (see e.g. Saha and Rao, 1980).

\subsection{Alternative Sub-Optimal Approaches}

Initial research on continuous-time model identification and estimation was not formulated in the above optimal manner but was based on the concept of a State Variable Filter (SVF) that generated the required prefiltered derivatives (see section 2.2.1 below). A comprehensive survey of these techniques has been given by Young (1981) and then by Unbehauen and Rao (1987, 1990, 1998) and Garnier et al. (2003). A book has also been devoted to these direct methods (Sinha and Rao, 1991). Most of the main, sub-optimal approaches are available in the CONtinuous-Time System IDentification (CONTSID) toolbox for Matlab ${ }^{\mathrm{TM}}$ (Garnier and Mensler 2000; Garnier et al. 2003), which also contains a version of the SRIVC algorithm. Since the methods have been documented so fully, however, it will suffice here merely to outline the main features of each approach.

\footnotetext{
${ }^{4}$ Note, however, that continuous-time noise modeling has been considered for models with no input $u(t)$ (Tuan 1977; Fan et al. 1999; Pham 2000; Söderström and Mossberg 2000; Larsson 2004); and some extensions have been made to handle the case of continuous-time ARX models (Söderström et al. 1997).
} 


\subsubsection{State-Variable Filter (SVF) Methods}

These methods originated from the first author's early research in this area (Young 1964, 1965a,b, 1970) and was referred to as the Method of Multiple Filters (MMF). It involves passing the input and output signals through a chain of (usually identical) first order prefilters with user-specified bandpass, normally selected so that it spans the anticipated bandpass of the system being identified. More recently this MMF approach has been re-named the Generalized Poisson Moment Functionals (GPMF) approach (Saha and Rao 1983; Unbehauen and Rao 1987). Recent MMF/GPMF developments have been proposed by the second author and his co-workers (Garnier et al. 19941995 1997 2000; Bastogne et al. 2001).

\subsubsection{Integration-Based Methods}

The main idea of these methods is to avoid the differentiation of the data by performing an order $n$ integration. These integration-based methods can be roughly divided into two groups. The first group, using numerical integration and orthogonal function methods, performs a basic integration of the data and special attention has to be paid to the initial condition issue. The second group includes the Linear Integral Filter (LIF: Sagara and Zhao 1990) and the Reinitialized Partial Moments (RPM: Jemni and Trigeassou 1996) approaches. Here, advanced integration methods are used that avoid the initial condition problem either by exploiting a moving integration window (LIF) or a time-shifting window (RPM).

\subsubsection{Modulating Function Methods}

This approach was first suggested almost half a century ago by Shinbrot in order to estimate the parameters of linear and nonlinear systems (Shinbrot 1957). Further developments have been based on different modulating functions. These include the Fourier-based functions (Pearson et al. 1994), in either trigonometric or complex exponential form; spline-type functions; Hermite functions and, more recently, Hartley-based functions (Unbehauen and Rao 1998). A very important advantage of using Fourier- and Hartley-based modulating functions is that the model estimation can be formulated entirely in the frequency domain, making it possible to use efficient Fast Fourier Transform (DFT/FFT) techniques. 


\subsection{The Advantages of Direct Continuous-Time Estimation}

Direct continuous-time model identification and estimation is advantageous for a number of reasons but four of these have particular practical importance. First, most scientific laws used in scientific model formulation, such as mass and energy conservation, are more naturally formulated in continuous-time differential equation terms. Second, while discrete-time models have different parameter values, dependent upon the sampling interval of the data, continuous-time models are defined by a unique set of parameters that are independent of the sampling interval. Third the direct continuous-time methods can be adapted easily to handle the case of irregularly sampled data. Finally, and perhaps most importantly, continuous-time models can be identified and estimated from rapidly sampled data, whereas discrete-time models encounter difficulties when the sampling frequency is too high in relation to the dominant frequencies of the system under study (Aström 1969). In this situation, the eigenvalues lie too close to the unit circle in the complex domain and the discrete-time model parameter estimates become statistically ill-defined. The practical consequences of this are either that the discrete-time estimation fails to converge properly, so providing an erroneous explanation of the data; or that even if convergence is achieved, the continuous-time model, as obtained by standard conversion (e.g. the Matlab ${ }^{\mathrm{TM}}$ D2CM function) from the estimated discrete-time model, does not provide the correct continuous-time model.

In order to illustrate the robustness of continuous-time estimation methods to the sampling interval of the data, a Monte Carlo Simulation (MCS) study was carried out (Young 2004) based on 50 stochastic realizations of a simulated effective rainfall-flow model, with the data sampled at sampling intervals from 5 minutes to 24 hours. Independent white noise (at a $20 \%$ level by standard deviation) was added to the simulated output for each realization. Only 50 realizations were used since the MCS in this case is computationally very intensive, with sample sizes ranging from 52, 128 to 181 . The SRIVC results obtained in this manner were compared with the estimation results obtained from the same data using two, indirect, discrete-time estimation methods: namely the RIV method mentioned previously and the Prediction Error Minimization (PEM) method available in the Matlab ${ }^{\mathrm{TM}}$ System Identification Toolbox. For each realization, the estimation was designated a failure if the error on a parameter estimate was greater than three standard deviations from the true value. This satisfactorily detected all convergence failures (where the estimates were always far from the true values, much greater than three standard deviations), without misclassification of any realizations.

It is clear from these MCS results that direct continuous-time model identification using the 
SRIVC algorithm is much more reliable than either of the indirect discrete-time methods considered. In particular, the direct continuous-time identification has no failures for sampling intervals up to one hour and only $0.32 \%$ thereafter. By contrast, the RIV-based indirect method has mean failure rates at short, medium and long sampling intervals of $7.1 \%, 2.5 \%$ and $1.5 \%$, respectively; while the equivalent figures for the PEM-based indirect method are 8.2\% 6.3\% and $11.5 \%$. The main reason for the rather poorer performance of the PEM-based indirect approach appears to be because the rainfall-flow system is 'stiff', being characterized by widely spaced eigenvalues (similar to the situation with the solute transport model described in the next section). This makes the PEM-based gradient optimization algorithm sensitive to the initial estimates and can result in convergence to non-global minima (see e.g. Ljung 2003). In other MCS studies using heteroscedastic additive noise, similar to that encountered on real flow data, the SRIVC results remain excellent but the indirect estimation results are worse than those reported here, with mean failure rates of the PEM-based indirect method of up to $45 \%$ (Young 2004) $)^{5}$.

\subsection{Linear Example: Pollutant Transport in River Systems}

Both of the practical examples described in this paper are examples of Data-Based Mechanistic (DBM) modelling (see Young 1998 and the prior references therein). This can be contrasted with 'black-box' modelling, since DBM models are only deemed credible if, in addition to explaining the time series data in a statistically efficient, parsimonious manner, they also provide an acceptable physical interpretation of the system under study. They can also be contrasted with 'grey-box' models, because the model structure is inferred inductively from the data, rather than being assumed a priori before model identification and estimation in a hypothetico-deductive manner (see the discussion in Young 2002a).

This first DBM modelling example is based on the analysis of data obtained from a tracer experiment in a river system. Tracer experiments ${ }^{6}$ are an excellent way of evaluating how a river transports and disperses a dissolved, conservative pollutant (solute). Figure 1 shows a typical set of tracer data from the River Conder, near Lancaster in North West England. This river is fairly small, with a cobbled bed, and the experiment involved the injection of $199 \mathrm{mg}$ of the dye tracer

\footnotetext{
${ }^{5}$ All of these estimation results were computed in version 6.5 of Matlab ${ }^{\mathrm{TM}}$ using version 5.0.2 of the Systems IDentification Toolbox (SID) and CAPTAIN toolboxes. The PEM-based results were quite a lot worse when using version 4 of the Matlab ${ }^{\text {TM }}$ SID toolbox, which utilizes a previous version of PEM.

${ }^{6}$ The interested reader will find a more complex example of ADZ modelling in Young (2001b), where the same approach used here is applied to data from a tracer experiment conducted in a large Florida wetland area.
} 
Rhodamine WT, with the measurement locations situated 400 metres apart, some way downstream of the injection location to allow for initial mixing. The river flow rate was measured at $1.3 \mathrm{~m}^{3} / \mathrm{sec}$.

The best known TF model for solute transport and dispersion is the Aggregated Dead Zone (ADZ) model introduced by Beer and Young (1983). It has become conventional to identify and estimate this model in discrete-time $\mathrm{TF}$ form and then deduce the continuous-time (differential equation) model parameters from the estimated parameters of this discrete-time TF (the indirect method). More recently, however, in related research on imperfect mixing processes (Price et al. 1999), continuous-time models have proven more useful. Moreover, in the present example, discrete-time modelling is not very successful when applied to the data in Figure 1: using a relatively fast sampling interval of 0.25 minutes, both the discrete-time RIV algorithm and the alternative PEM algorithm yield second order models which do not explain the data very well. Moreover, while these algorithms produce well fitting third order models, these are clearly over-parameterized and have complex roots, so that the models can be rejected on DBM grounds since they have no obvious physical interpretation.

Continuous-time SRIVC modelling of the tracer data is much more successful and also produces models that can be interpreted directly in physically meaningful terms, so satisfying the DBM modelling requirements. This suggests strongly that the dynamic relationship between the measured concentrations at the input (upstream) and at the output (downstream) measurement sites is linear and second order, with the continuous-time TF identified by the SRIVC algorithm in the following form

$$
y(t)=\frac{b_{0} s+b_{1}}{s^{2}+a_{1} s+a_{2}} u(t-\tau)+e(t)
$$

or, in ordinary differential equation terms,

$$
\frac{d^{2} y(t)}{d t^{2}}+a_{1} \frac{d y(t)}{d t}+a_{2} y(t)=b_{0} \frac{d u(t-\tau)}{d t}+b_{1} u(t-\tau)+\mu(t)
$$

where $\mu(t)=\left(s^{2}+a_{1} s+a_{2}\right) e(t)$. Here, time is measured in minutes and the pure time delay of $\tau=3$ minutes on the input variable is the purely advective, 'plug flow' effect. There is very little serial correlation and some heteroscedasticity in the estimated residuals, but the variance is extremely low (0.0001), as reflected in the very high coefficient of determination based on these modelling errors of $R_{T}^{2}=0.9984$ (i.e. $99.84 \%$ of the measured output variance is explained by the simulated output of the model $)^{7}$. Note that it is this extremely low variance and near whiteness of the model residuals that justifies our use of the SRIVC algorithm in this case: not only are the parameter estimates asymptotically unbiased because of the low noise level and the use of the IV approach in

\footnotetext{
${ }^{7} R_{T}^{2}$ is defined as $R_{T}^{2}=1-\operatorname{var}\{y(t)-\hat{x}(t)\} / \operatorname{var}\{y(t)\}$, where $\hat{x}(t)$ is the deterministic model output from (14).
} 
SRIVC, but the estimated standard error bounds provide a good indication of the uncertainty in these estimates.

With such a high $R_{T}^{2}$, the model (12) obviously explains the data very well, as shown in Figure 2 which compares the deterministic (noise free) model output

$$
\hat{x}(t)=\frac{\hat{b}_{0} s+\hat{b}_{1}}{s^{2}+\hat{a}_{1} s+\hat{a}_{2}} u(t-3)
$$

with the measured tracer concentrations $y(t)$. The estimated parameters are as follows:

$$
\begin{array}{ll}
\hat{a}_{1}=2.0513(0.073) ; & \hat{a}_{2}=0.6032(0.055) \\
\hat{b}_{0}=1.1939(0.014) ; & \hat{b}_{1}=0.6428(0.056)
\end{array}
$$

where the figures in parentheses are the estimated standard errors. Introducing the estimated parameter values, the TF model (14) can be decomposed by partial fraction expansion into the following form

$$
\hat{x}(t)=\frac{0.6081}{1+0.5898 s} u(t-3)+\frac{0.4575}{1+2.8105 s} u(t-3)
$$

which reveals that the model can be considered as a parallel configuration of two first order processes which appear to characterize distinctive solute 'pathways' in the system with quite different residence times: one 'quick', with a residence time $T_{q}=0.5898$ minutes; and the other 'slow', with a residence time $T_{s}=2.8105$ minutes. The associated steady state gains are $G_{q}=0.6081$ and $G_{s}=0.4575$, respectively. These suggest a parallel partitioning of tracer with a partition percentage of $P_{q}=100[0.6081 /(0.6081+0.4575)]=57.1 \%$ for the quick pathway, and $P_{q}=100[0.4575 /(0.6081+0.4575)]=42.9 \%$ for the 'slow' pathway. As expected, the sum of the estimated steady state gains for the two pathways (1.0656) is equal to the total estimated steady state gain of the complete TF model (0.6428/0.6032), which has an estimated standard error of 0.1658 . These figures can be compared with the ratio of the areas under the input and output signal graphs, which is 1.086 , and the gain of unity that, in a perfect experiment with no loss/gain of tracer or measurement errors, corresponds to the complete conservation of the tracer mass. In this regard, it is clear that, taking into account the estimated uncertainty, the estimated gain insignificantly different from unity.

The decomposition of the TF into the parallel pathway form (17), provides the information required to interpret the model in a simple physically meaningful manner. The first order model associated with each pathway can be considered as a differential equation describing mass conservation (see e.g. Wallis et al., 1989). And if it is assumed that the flow is partitioned in the same way as the dye, then the Active Mixing Volume (AMV: see Young and Lees 1993) of water 
associated with the dispersion of the solute in each pathway can be evaluated by reference to equation (17), the flow rate and the residence times. This yields a quick pathway AMV, $V_{q}=26.3 m^{3}$; and a slow pathway AMV, $V_{s}=94.1 \mathrm{~m}^{3}$, respectively. The associated Dispersive Fraction (DF), in each case, is calculated as the ratio of the AMV and the total volume of water in the reach, giving $D F_{q}=0.12$ and $D F_{s}=0.56$ : (i.e. the active mixing volumes are $12 \%$ and $56 \%$ of the total volume of water in each pathway, respectively). In other words, the slow pathway results in a considerably greater dispersion (and longer-term detention) of the dye than the quick pathway, as one might expect. While the model, interpreted in this simple manner, provides little insight into the detailed, complex processes of solute transport and dispersion in the river, it does provide a meaningful explanation of the overall, aggregative behaviour and the dominant pathways in the system. Such an explanation is clearly appropriate in applications related to the monitoring and control of water quality in the river system at this aggregative level

Given this quantitative analysis of the model (14), the most obvious physical interpretation of the parallel flow decomposition in (17) is a form of two layer flow, with the slow pathway representing the dye in the water moving adjacent to the cobbled bed and banks of the river, which is being differentially delayed in relation to the quick pathway, which is associated with the more freely moving surface layers of water. The aggregated effect of each pathway is then an advective transportation delay of 3 minutes, associated with the non-dispersive advection ('plug flow'); and an ADZ, defined by the associated AMVs and DFs in each case, which are the main mechanisms for dispersion of the dye (and, therefore, other forms of pollution) in its passage down the river.

This parallel partitioning of the flow and solute also helps to explain the shape of the experimentally measured concentration profile. The individual concentration profiles for the quick and slow pathways, as inferred from the parallel partitioning, are shown as dashed and dash-dot curves, respectively, in Figure 2.

\section{Nonlinear Continuous-Time Model Identification}

The identification and estimation of nonlinear continuous-time models is considerably more difficult than linear modelling. First, there is no unified theory for nonlinear systems and so it is necessary to consider a given 'class' of nonlinear model. Secondly, the estimation of time derivatives is more difficult because the commutation operation that is so important in defining prefiltered timederivatives (see section 2.1) is no longer possible in the case of nonlinear systems. Here, we consider the State Dependent Parameter (SDP) class of nonlinear models which can describe a wide variety 
of nonlinear systems including chaotic processes.

\subsection{State Dependent Parameter Estimation}

As far as the authors are aware, the idea of State Dependent Parameter (SDP) modelling within a stochastic setting was originated by Young (1969a,b) and Mendel (1969). They enhanced recursive estimation performance by assuming that the model parameters could vary over time because of their dependence on the variations in other measured variables. These ideas were then explored within a broader SDP setting (Young 1978) and Priestley (1988) took them up in a series of papers and a book on the subject. These earlier publications do not, however, exploit the power of recursive Fixed Interval Smoothing (FIS), which provides the main engine for the latest methods of SDP estimation (see Young et al. 1999; Young 2000 2001a; Young et al. 2001).

There is some similarity between SDP models and Linear Parameter Varying (LPV) models, as pointed out in Young (2005) in a comment on the paper by Previdi and Lovera (2004). However, there are significant differences. For instance, while the LPV approach tends to be a fully parametric, black-box method, SDP estimation is a combination of non-parametric and parametric estimation aimed at opening up this black-box model and, if at all possible, explaining it in physically meaningful terms. In particular, SDP modelling exploits recursive FIS estimation in an initial structure identification stage of the modelling in order to obtain the location of nonlinearities in the model, together with non-parametric (graphical) estimates of how the SDPs are related to the state on which they are dependent. This information then forms the basis for the parameterization of the nonlinearities and the final estimation of this parametric DBM model (see the later example in section 3.4).

SDP estimation was originally developed in discrete-time terms (see above references). The simplest SDP continuous-time model is a nonlinear equivalent of the linear TF model (1) and takes the following form

$$
y(t)=\frac{B\left(s, \mathbf{z}_{t}\right)}{A\left(s, \mathbf{z}_{t}\right)} u(t-\tau)+e(t)
$$

where $A\left(s, \mathbf{z}_{t}\right)$ and $B\left(s, \mathbf{z}_{t}\right)$ are SDP polynomials in the $s$ operator of the form

$$
\begin{aligned}
& A\left(s, \mathbf{z}_{t}\right)=s^{n}+a_{1}\left(z_{1, t}\right) s^{n-1}+\cdots+a_{n}\left(z_{n, t}\right) \\
& B\left(s, \mathbf{z}_{t}\right)=b_{0}\left(z_{n+1, t}\right) s^{m}+\cdots+b_{m}\left(z_{n+m+1, t}\right)
\end{aligned}
$$

while $\mathbf{z}_{t}$ is a vector of measured variables (states) on which the parameters may be dependent. In 
estimation equation terms, this model can be written most conveniently as:

$$
s^{n} y(t)=\mathbf{z}_{t}^{T} \mathbf{p}_{t}+e_{t}
$$

where,

$$
\begin{aligned}
& \mathbf{z}_{t}^{T}=\left[-s^{n-1} y(t) \ldots-y(t) s^{m} u(t-\tau) \ldots u(t-\tau)\right] \\
& \mathbf{p}_{t}=\left[p_{1}\left(z_{1, t}\right) \ldots p_{n+m+1}\left(z_{n+m+1, t}\right)\right]^{T}
\end{aligned}
$$

while,

$$
p_{1}\left(z_{1, t}\right)=a_{1}\left(z_{1, t}\right) ; p_{2}\left(z_{2, t}\right)=a_{2}\left(z_{2, t}\right) \cdots, p_{n+m+1, t}\left(z_{n+m+1, t}\right)=b_{m}\left(z_{n+m+1, t}\right)
$$

Here, $p_{i}\left(z_{i, t}\right)$ indicates that the $i^{\text {th }}$ parameter varies over time as an (initially unknown) function of another measured variable $z_{i, t}$ which could be any measured variable but is quite often the input variable $u(t)$, the output variable $y(t)$ or functions of these variables (e.g. time derivatives, lagged values etc.).

Although the SDP model (18) has the superficial appearance of a linear, time variable parameter TF model, this is quite deceptive since the model represents a widely applicable class of truly nonlinear systems. This becomes apparent when it is noted that the time variable parameters $a_{i}\left(z_{i, t}\right)$ in this model are not slowly variable, as in the case of standard TVP models, they are functions of the variables $z_{i, t}$ that relate directly to the state of the system. As a result, they can vary very rapidly: indeed, in the case of nonlinear chaotic systems, they will also vary chaotically. This means that the SDP model does not simply represent a system in a limited neighbourhood of some defined reference state, it can represent wide-ranging and truly nonlinear behaviour. So, although they cannot represent every type of nonlinear stochastic-dynamic behaviour, such SDP models (which can be extended to include multivariate state dependency with each SDP a function of more than one state variable) can represent a very wide variety of nonlinear systems, as shown in the references cited above. For this reason, they seem to have great potential within a general environmental context.

As mentioned above, SDP modelling consists of two stages, the full details of which are given in the above references. In the first, non-parametric stage, the recursive SDP estimation algorithm is an extension of the stochastic approach to Time Variable Parameter (TVP) estimation, where the time variations in the parameters are assumed to evolve as one of the Generalized Random Walk (GRW) class of Gauss-Markov process (e.g. Young 1999), of which the Random Walk (RW) and Integrated Random Walk (IRW) processes are the best known. However, SDP estimation differs from standard TVP estimation in three important respects. First, by exploiting the forward and 
backward approach of recursive smoothing (FIS) estimation rather than forward 'filtering' approach used in most standard TVP estimation, it is able to obtain lag-free and statistically more efficient estimates of changing parameters, so enhancing the ability to interpret these parametric variations in SDP terms. Second, in order to allow for the rapid variation that state dependency can induce in the parameters, the data are sorted into some other, non-temporal order (e.g. ascending order of magnitude), so that the rate of change of the parameter variations between samples in this sorted data space is much smaller than in the original observation space. Thirdly, in order to allow for the possibility of different state dependency in each parameter, an iterative 'back-fitting' algorithm is used to estimate each SDP separately, based on prior estimates of all the other SDPs in the model. The main estimation engine used in the implementation of this back-fitting algorithm is FIS estimation.

As in the linear situation, the main problem with the above SDP estimation methodology in the continuous-time case is its requirement for measurements of the input and output time derivatives

$$
\begin{gathered}
s^{i} y(t)=\frac{d^{i} y(t)}{d t^{i}}, \quad i=1,2, \ldots, n \\
s^{j} u(t-\tau)=\frac{d^{j} u(t-\tau)}{d t^{j}}, \quad j=1,2, \ldots, m
\end{gathered}
$$

which are not usually available directly. The approach to this time derivative estimation problem used in the later example (section 3.4) is again based on recursive FIS estimation (Young et al. 1993; Young 1993). Each variable in question (here $u(t)$ or $y(t))$ is modelled as a multiple differentiation process in continuous-time and then converted to the discrete-time equivalent of this process. The time derivatives of the variable at the sampling instants are then recovered as the FIS estimated states of this model. In the simplest, single derivative case used in the example discussed later in section 3.4, for instance, this takes the form of an IRW process with its parameter defined by the sampling interval, $\Delta t$. This process could be formulated more accurately in continuous-time terms but the discrete-time formulation works quite well, as we shall see in the later example (section 3.4; see also the above references). Note also that this estimation of time derivatives is only required in the first, non-parametric stage of SDP estimation. The subsequent, final parametric estimation stage, as discussed below, involves direct integration of the differential equation and some form of optimal or sub-optimal estimation, so that no explicit derivative estimation is required. 


\subsection{Final Parametric Estimation}

Within DBM modelling, non-parametric SDP modelling normally provides a method for identifying the presence, location and nature of nonlinearities in the SDP models. In this manner, it serves as a prelude to the parameterization of the final nonlinear stochastic model and the more efficient estimation of the (normally constant) parameters that characterize this nonlinear parametric model. Such estimation can be based on various approaches to model optimization, from nonlinear least squares, through maximum likelihood and optimal instrumental variable estimation, to the latest Monte Carlo-based methods of Bayesian estimation. But the methodology used in any particular application will normally depend on the nature of the system under study, the modelling objectives, and the scientific background of the model builder. In the case of the global carbon cycle example discussed below, optimization is based again on a Prediction Error Minimization (PEM) approach (maximum likelihood estimation in the case of Gaussian residuals).

\subsection{Other models}

Although we have outlined the SDP approach to data-based nonlinear model identification and estimation in terms of continuous-time transfer function models, SDP estimation is not restricted to such models. It can be applied to any continuous or discrete-time, nonlinear stochastic model that can be formulated as linear additive sum of nonlinear elements (sometimes termed an 'affine' model). This ranges from static nonlinear regression models (Young 2001b) to dynamic state space models. Typical examples of the latter are the well known Lorenz Strange Attractor (Young 1993), the model of the Nicholson Blowfly Data (Young 2000) and the simpler global carbon cycle model considered below.

\subsection{Global Carbon Cycle Modelling}

This example is a summary of much more comprehesive results obtained in a recent study of the global carbon cycle, a complete description of which is given in Young and Jarvis (2002). In particular, it investigates the dynamic relationship, over the period 1856 to 2000, between globally averaged annual measures of $\mathrm{CO}_{2}$ emissions (arising from both the use of carbon fuels and land-use changes); perturbations in atmospheric carbon dioxide partial pressure, $\mathrm{pCO}_{2}$; and the Northern Hemisphere temperature anomaly. The analysis reveals the possible presence of a temperaturedependent nonlinearity in the dynamic relationship between $\mathrm{CO}_{2}$ emissions and atmospheric $p \mathrm{CO}_{2}$ that has an interesting and potentially important physical interpretation. 
Figure 3 presents the data used in the analysis and shows that there are clear increases in all three variables over the period from 1856 to the end of the last century. The normal statistical procedure would be to reduce the series to stationarity in some manner (e.g. by differencing). But the climate data have a clear physical meaning and we can be reasonably sure that the increase in the levels of atmospheric $\mathrm{pCO}_{2}$ are the result of the increases in emissions. In other words, there is an obvious input-output relationship, with the non-stationarity in the input giving rise to non-stationarity in the output. What is much less certain, however, is that the rise in the level of the atmospheric $\mathrm{pCO}_{2}$ is, in turn, leading to the observed increase in the temperature anomaly (see later in section 3.4.4).

With these factors in mind, we will analyze the data directly in the form shown in Figure 3. For this analysis, the input $\mathrm{CO}_{2}$ emissions, the output atmospheric $\mathrm{pCO}_{2}$ perturbations about the assumed pre-industrial level (see caption to Figure 3), and the temperature anomaly will be denoted by $u(t), y(t)$ and $T(t)$, respectively.

\subsubsection{Linear model identification and estimation}

The SRIVC algorithm identifies a number of linear, constant parameter, first order models that have good identification and estimation statistics; i.e. coefficients of determination $R_{T}^{2}$ based on the simulated output greater than 0.99 (i.e. the simulated deterministic output of the model explains $>99 \%$ of the $\mathrm{pCO}_{2}$ variance), together with well defined, low standard error parameter estimates. However, all estimated higher order models are rejected, either because they do not satisfy these statistical criteria or because they are not satisfactory in dynamic terms (e.g. they have unstable or imaginary eigenvalues).

Two important factors emerge from this analysis: first, it is necessary to add a small additional constant input to obtain a good explanation of the $y(t)$ series (see below); second, there is a less well-defined pure time delay of about 5 years. In other words, this initial analysis suggests a model of the form:

$$
\begin{cases}\frac{d x(t)}{d t} & =a_{1} x(t)+b_{0} u(t-\tau)+c \\ y(t) & =x(t)+\xi(t)\end{cases}
$$

where $x(t)$ is the underlying, 'noise-free' $p \mathrm{CO}_{2}$ perturbation; $\tau$ is the pure time delay; $\xi(t)$ is the residual coloured noise at the output of the model, with zero mean value and variance $\sigma_{\xi}^{2}$; and $c$ is an additional constant input. The addition of $c$ makes sense physically because it will account for the small dynamic effects of anthopogenic emissions pre-1856, as well as correcting for any small 
inaccuracy in either the assumed pre-industrial level of $\mathrm{pCO}_{2}$ that has been removed from the $p \mathrm{CO}_{2}$ data (see caption to Figure 3), or the $\mathrm{CO}_{2}$ emissions

In order to obtain an improved, possibly non-integer, estimate of the time delay, the model (27) was estimated using the leastsq optimization tool in Matlab ${ }^{\mathrm{TM}}$, with the model simulated in Simulink ${ }^{\mathrm{TM}}$, using the linsim tool. All integrations of the model used in this optimization were initiated from the measured $y(0)$, since separate optimization of the initial condition had little effect on the estimates. Also, in order to allow for colour in the residuals $\xi(t)$, they were modelled as a discrete-time AR process, i.e.

$$
\xi_{t}=\frac{1}{1+d_{1} z^{-1}+\ldots+d_{n} z^{-n}} e_{t}
$$

where $z^{-r}, r=1,2, \ldots n$ is the backward shift operator; while $\xi_{t}=\xi\left(t_{i}\right)$ and $e_{t}=e\left(t_{i}\right)$ are, respectively, $\xi(t)$ and $e(t)$ sampled at the annual sample times $t_{i}, i=1,2, \ldots, N$, and $N$ is the sample size. The sampled stochastic model residuals $e_{t}$ are assumed to be a zero mean sequence of serially uncorrelated random variables with variance $\sigma^{2}$ (discrete white noise). This 'hybrid' approach to modelling was used because the discrete-time stochastic model (28) is easier to handle within the optimization (and in theory). In any case, the noise model estimation makes only a small difference to the final estimated parameter values because the residual noise variance is so small (although it does affect their estimated uncertainty).

Parameter estimation was based on optimization of the following least squares PEM cost function with respect to the unknown parameters

$$
J\{\boldsymbol{\theta}\}=\sum_{t=1}^{t=N} \hat{e}_{t}^{2}, \quad \boldsymbol{\theta}=\left[\begin{array}{lllllll}
a_{1} & b_{0} & c & d_{1} & d_{2} & \ldots & d_{n}
\end{array}\right]^{T}
$$

where $\hat{e}_{t}$ are the stochastic model residuals (one-year-ahead prediction errors) at the annual sampling interval. The estimated parameters in the most important continuous-time part of the model, based initially on the whole data set $(N=145)$ are as follows:

$$
\begin{aligned}
& \hat{a}_{1}=-0.0167(0.0009) ; \quad \hat{b}_{0}=0.0371(0.0009) ; \\
& \hat{c}=0.0114(0.0006) ; \quad \hat{\tau}=5.0(0.389) ; \\
& \sigma_{\xi}^{2}=0.0041 ; \quad \sigma^{2}=0.0004
\end{aligned}
$$

where, once again, the numbers in parentheses are the estimated standard errors. The noise model (28) is identified by the Akaike Identification Criterion (AIC: Akaike, 1974) as either an AR(8) or $\operatorname{ARMA}(2,2)$ process, with the final residuals $e_{t}$ showing no significant auto-correlation and no 
cross-correlation with the input $u_{t}$ (the annual samples of the input $\mathrm{CO}_{2}$ emissions). However, the residuals $\hat{\xi}_{t}=y_{t}-\hat{x}_{t}$, although very small, are significantly correlated with the temperature $T_{t}$ for all lags between 4 and 22 years and a maximum correlation coefficient of $0.4(0.17)$ at a lag of 10 years. Moreover, recursive FIS estimation of the local cross correlation is much larger than this at high values of $T_{t}$. The model has a coefficient of determination based on the error $\hat{\xi}_{t}$ between the simulated deterministic model output and the measured $\mathrm{CO}_{2}$ of $R_{T}^{2}=0.9991$; while the more conventional coefficient of determination based on the one-step-ahead prediction errors $\hat{e}_{t}$ is $R^{2}=0.9999$. This is, of course, important in the present context since it means that the main differential equation which, as we shall see below has some physical significance, is explaining the measured output of the dynamic system very well indeed in both simulation and prediction terms.

\subsubsection{Nonlinear model identification and estimation}

Despite the apparently very good results obtained in linear modelling, the high correlation of the residuals $\hat{\xi}_{t}$ with the temperature anomaly $T_{t}$ suggests that there may be a temperature dependent component in the model residuals that is capable of being absorbed within the model and so improving its descriptive ability still further. This is indeed the case: SDP non-parametric estimation suggests strongly that, while there is no evidence that the input parameter $b_{0}$ is time variable, the parameter estimate $\hat{a}_{1}$ seems to vary significantly as a function of the temperature anomaly $T_{t}$. This SDP estimate $\hat{a}_{1}\left(T_{t}\right)$, plotted as the dash-dot line in the left hand panel of Figure 4, suggests that the parameter value is becoming more negative as the temperature increases, although there is a tendency for the variations to 'flatten out' at the lower and upper extremes of the temperature anomaly range. But we must remember that these are non-parametric estimates and tend to be more poorly defined in these regions because of end effects and the paucity of data in these regions.

In order to investigate this problem in a statistically more efficient parametric manner, several different parameterizations of the temperature dependency suggested in Figure 4 were tried, including polynomial, radial basis function (e.g. Ordieres et al., 2005) and an exponential decline. However, the best results were obtained with either simple linear (first order polynomial) or sigmoidal $^{8}$ laws in $T(t)$. In other words, the finally identified nonlinear model takes either of the

\footnotetext{
${ }^{8}$ This kind of nonlinearity has also been termed a 'two-regime, smooth transition threshold nonlinearity' (Granger and Teräsvirta 1993)
} 
following forms

$$
\left\{\begin{array}{l}
\frac{d x(t)}{d t}=\{\alpha+\beta T(t)\} x(t)+b_{0} u(t-\tau)+c \\
y(t)=x(t)+\xi(t)
\end{array}\right.
$$

or

$$
\left\{\begin{array}{l}
\frac{d x(t)}{d t}=\left\{\alpha+\frac{1}{1+e^{-\beta T(t)}}(\gamma-\alpha)\right\} x(t)+b_{0} u(t-\tau)+c \\
y(t)=x(t)+\xi(t) .
\end{array}\right.
$$

In the case of (34), the estimated parameters, again based initially on the whole data set $(N=145)$, are as follows ${ }^{9}$

$$
\begin{aligned}
& \hat{\alpha}=-0.0232(0.0062) ; \hat{\beta}=-4.503(7.321) ; \hat{\gamma}=-0.0128(0.0054) ; \\
& \hat{b}_{0}=0.0402(0.0017) ; c=0.0066(0.0015) ; \\
& \hat{\tau}=5.0 ; \sigma_{\xi}^{2}=0.00240 ; \sigma^{2}=0.000623 \\
& R_{T}^{2}=0.99949, \quad R^{2}=0.99987
\end{aligned}
$$

where the $\mathrm{AIC}$ once more identifies an $\operatorname{AR}(8)$ or $\operatorname{ARMA}(2,2)$ model for the noise $\xi_{t}$. Also as before, the final model residuals $e_{t}$ show no significant auto-correlation and no cross-correlation with the input $u_{t}$. But now $\hat{\xi}_{t}$ also shows no significant correlation with $T_{t}$ (statistically insignificant maximum correlation of $0.11(0.17)$ at a lag of 10 years), as required. Bearing on our earlier discussion, note that the estimate of $c$ is significant but very small, as anticipated.

The sigmoidal law obtained from the above estimation of model (34) is shown as the full line in the right hand panel of Figure 4. This sigmoidal law was introduced in order to allow for any possible flattening out of the relationship at higher levels of the temperature anomaly (see earlier discussion) which would have most effect on long-term model predictions. This model explains the data marginally better than the linear-in-temperature model (33) and, while its $\beta$ parameter is rather poorly defined statistically, this is quite normal for nonlinearities of this type (Granger and Teräsvirta 1993). For comparison, the estimated linear-in-temperature law is shown as the full line in the left hand panel of Figure 4.

Finally, although the models (33) and (34) have been identified and estimated in a largely 'blackbox' manner, they can both be interpreted in physically meaningful terms at the global scale, as required by the DBM approach to modelling. A full interpretation of the models in this regard is provided in Young and Jarvis (2002). It is sufficient here to say that they can be considered to

\footnotetext{
${ }^{9}$ These results were obtained with a fixed $\tau=5$ : however, this was based on prior estimation with $\tau$ allowed to take on non-integer values.
} 
represent the mixing dynamics of anthropogenic $\mathrm{CO}_{2}$ emissions within the active elements of the global carbon cycle. Both models yield similar results in this regard. In 1980, the total mass of $\mathrm{CO}_{2}$ in this identified mixing system is estimated as $842.9(30.2) G t$ : this is somewhat larger than the quoted figures for the mass of $\mathrm{CO}_{2}$ in the atmosphere alone in 1980, which is given in the literature as 730Gt (Farquhar et al. 2001), suggesting that, as might be expected since they are based on globally averaged data, our models include the mixing effects of other surface ocean and terrestrial carbon reservoirs that interact with the atmospheric system. Also, for the period 1950-1984, the cumulative loss of $\mathrm{CO}_{2}$ attributable to the nonlinear negative feedback effect that we have identified is 13.5 (1.8) Gt, which is comparable to the $20(5) G t$ estimated by Dai and Fung (1993) using a completely different approach. The estimated total climatic losses for the period 1856-2000 are 39.4(6.2)Gt. This may provide a credible explanation for some of the so called 'missing sink' for $\mathrm{CO}_{2}$ that is often cited in the literature (Farquhar et al. 2001).

\subsubsection{Predictive validation}

In order to investigate the predictive capacity of the model (34), it is re-estimated on the basis of only the first 120 annual samples up to 1975 and then its performance is evaluated by forecasting the perturbational $\mathrm{pCO}_{2}$ variations over the last 25 years of the $20^{\text {th }}$ Century, without any reestimation of the parameters over this period. To make this exercise more demanding, the model integrations are initiated in 1856, with the initial condition for the integrations set to the value of $y(0)=0.6523 \mathrm{pa}$ on this date and with no reference to the actual $y(t)$ measurements at all after this. In other words, the forecast is based on a straightforward Monte Carlo Simulation (MCS) of the nonlinear model from this initial condition using only the measured $\mathrm{CO}_{2}$ emissions and temperature anomaly as inputs to the model. This MCS analysis is based on the estimated covariance matrix of the model parameters and involves 1000 random realizations of the model. The results are shown in Figure 5, where we see that the $y(t)$ variations are predicted very well, with $R_{T}^{2}=0.99941$ (almost the same as that obtained from model estimation based on all 145 samples) and the MCS estimated confidence region is small. For comparison, the linear-in-temperature model (33) performed marginally worse in predictive validation terms, with $R_{T}^{2}=0.99935$; while the linear model (27) was significantly worse (in statistical terms), with $R_{T}^{2}=0.99909$. 


\subsubsection{A controlled future?}

The simple, stochastic, nonlinear models discussed in the previous section could form the basis for long-term predictions of $\mathrm{pCO}_{2}$ and, when combined with an associated $p C \mathrm{O}_{2}$-global temperature model, could form the basis for long term climate predictions. However, such long term predictions are highly uncertain whatever climate model is utilized and it is, perhaps, better to avoid such speculative exercises. In our opinion, a more sensible alternative is to consider how it is possible to formulate a $\mathrm{CO}_{2}$ emissions policy that is relatively insensitive to the models used in its derivation. One such approach of this type is described by Young and Jarvis (2002), who consider the specification of carbon emissions policy as a stochastic feedback control problem. They carry out such an exercise using the following simple hybrid (see earlier) model obtained by SRIVC and RIV analysis of the globally averaged $\mathrm{pCO}_{2}$ and global temperature anomaly data shown in Figure 3 :

$$
\begin{aligned}
\frac{d z(t)}{d t} & =a_{1} z(t)+b_{0}\{y(t)-\bar{y}\} \\
T_{t} & =z(t)+\eta(t)
\end{aligned}
$$

where $\bar{y}$ is the mean value of $y(t)$ (which is subtracted from $y(t)$ so that the input is a perturbational series, as in the case of the output temperature anomaly series, $T(t)) ; z(t)$ is the underlying 'noisefree' temperature anomaly; $\eta_{t}$ is an $\mathrm{AR}(9)$ process and the estimates of the two parameters in (35) are:

$$
\hat{a}_{1}=0.0514(0.009) ; \quad \hat{b}_{0}=0.0080(0.001)
$$

It must be stressed that we are not claiming that this is a good model for the effects of the perturbations in $\mathrm{pCO}_{2}$ on global energy balance: its descriptive ability is too limited in this regard $\left(R_{T}^{2}=0.56 ; R^{2}=0.72\right)$ and a more detailed analysis of this relationship is required. Rather it is being used here merely for illustrative purposes and to ensure that the scaling between $y(t)$ and $T(t)$ is of the correct order to ensure that the simulation of the complete feedback system makes reasonable sense. Although we do not feel that long term predictions based on this model, combined with the simple global $\mathrm{pCO}_{2}$ models discussed in previous sections, should be taken too seriously, they are quite interesting. For instance, as we see below, they suggest that any anthropogenicallyrelated future temperature rise might occur at a much slower rate than that predicted by the large, deterministic climate models that are normally used to make such predictions.

An important aspect of climate change studies is the definition of emission scenarios into the future that may control the global warming (under the hypothesis that a link between emissions and global temperature change is accepted). One approach is to consider the 'inverse problem' (see e.g. 
Wigley 2000): i.e. compute the emission scenarios that are able to achieve a range of 'stabilization concentration profiles' for atmospheric $\mathrm{CO}_{2}$, such as those utilized in the studies carried out by the IPCC (Schimel et al. 1996). An alternative and more robust approach is to exploit automatic control theory and so generate an emissions scenario (control input) that achieves some required objective, as defined by a specified 'optimal' criterion function.

However, if we consider the results obtained using approaches such as these, based on the models discussed in this paper, they are significantly different from those obtained heretofore by climate scientists. For example, in (Wigley 2000), a deterministic carbon balance model is used to generate a range of emission scenarios that achieve a whole range of stabilization concentration profiles, with steady $\mathrm{CO}_{2}$ levels ranging from 350 to 750 ppmv (i.e. perturbations in $\mathrm{pCO}_{2}$ from 7 to $47 \mathrm{pa}$ ). If we consider the 450 ppmv (17 pa) case, for instance, this deterministic analysis produces a future emissions scenario that reduces to a quasi-steady level of circa 2.3 Gt $y^{-1}$ by the year 2200 .

In contrast to the latter deterministic results, consider the stochastic simulation scenario presented in Figure 6. This shows the results of an automatic control exercise based on our most pessimistic linear model (27), which predicts the largest future rises in $\mathrm{pCO}_{2}$. In order to generate the results shown in Figure 6, the emissions input to the model is based on the measured emissions from 1856 to 2000. After this, however, the computed emissions input is generated by a Proportional-Integral-Plus (PIP) feedback controller (see Taylor et al. 2000 and the prior references therein) designed to follow a defined future $\mathrm{pCO}_{2}$ profile (in this case similar to that in Wigley (2000), but other profiles could be considered equally easily using this approach). This is designed to stabilize the atmospheric $\mathrm{pCO}_{2}$ perturbation at a level of 17.6pa (456ppmv) and the temperature anomaly at a mean level of circa $2.6^{\circ} \mathrm{C}$. Considering the results of this exercise shown in Figure 6 , we see that the objectives have been realized by 2200, with an emissions scenario stabilizing at a level of $7.4 G t y^{-1}$, with a $5 \%-95 \%$ percentile range between 6.1 and $8.5 G t y^{-1}$; i.e. between 3.6 to 6 Gt $y^{-1}$ higher than that computed in Wigley (2000) for a similar profile (i.e. the same result is achieved with a much smaller and more practically feasible reduction in the $\mathrm{CO}_{2}$ emissions).

One practical advantage of this automatic control solution, when compared with the inversion solution, is that the feedback control policy is applied incrementally, so that it is adjusted in relation to the measured data as time progresses. Thus if some disturbance to the system is experienced, the PIP controller will suggest adjustments to the $\mathrm{CO}_{2}$ emission policy that compensate for the disturbance and continue to follow the defined $\mathrm{pCO}_{2}$ profile as closely as possible without requiring excessive emission adjustments (as defined by the optimal control criterion function used in the PIP control system design). 
Of course, it must be emphasized that the above exercise is purely illustrative. Its main aim is to show how the continuous-time DBM model, as obtained in the manner described in this paper, might be useful in policy formation. Both the results in Wigley (2000) and ours are speculative 'what-if' studies, characterized by considerable uncertainty, even if it is assumed that the generated emissions scenario is achievable. They can also be considered as providing a range of extreme speculations, with the Wigley results representing a pessimistic future requiring major reductions in emissions; while ours are much more optimistic in this regard ${ }^{10}$. The credibility attached to these speculations will, of course, depend largely on one's scientific background. If we exaggerate the situation somewhat, in order to clarify the nature of the dilemma, then two extreme viewpoints can be discerned:

1. Scientists educated within a deterministic, hypothetico-deductive tradition, will argue correctly that a simulation model, such as that used by Wigley, reflects the current state-of-theart understanding of the mechanisms operative in those multiple compartments of the GCC system that are thought necessary to characterize its dynamic behaviour. They will point out that it is a representation that utilizes all of the information available in this regard, assembled in a model form that reflects the scientist's well-supported perception of how the different physical and biological processes interact with each other in dynamic terms. And they will emphasize that the level of detail in the model reflects a widely held view that the environment, particularly at the global scale, is a very complex system that can only be represented by a similarly complex model.

2. On the other hand, scientists who follow a statistically-based, inductive approach, of the kind employed in the present report, will argue that data-based, mechanistic models, identified and estimated in stochastic terms, better reflect the information content of the available, uncertain data; and that such models are less prone to the prior prejudice that can sometimes result from over-confidence in current paradigmatic judgment. They will also point out that large and complex models cannot be estimated properly in rigorous statistical terms, since they are over-parameterized and so not uniquely identifiable from the available data. As a result, they will argue that such a model has not been adequately validated against the available data and so is a questionable vehicle for making predictions into the distant future. And, finally, they will be able to show that, despite their complexity, large mathematical models behave in a

\footnotetext{
${ }^{10}$ although, ironically, they are based here on our most pessimistic, linear model: the nonlinear models would be even more optimistic because of the temperature feedback effects.
} 
quite simple dynamic manner and can be mimicked in this regard by much simpler 'dominant mode' mathematical models of the DBM kind (Young et al. 1996; Shackley et al. 1998) .

Both of these extreme viewpoints can be defended, of course, and the reader will not be surprised that we subscribe more to the latter, whilst strongly advocating continuing studies of all types that are aimed at better understanding climate system dynamics. But they are, nonetheless, rather dogmatic extremes: the most reasonable approach, in any given practical situation, probably lies somewhere between them. After all, there is never only one model of a real system and the choice between them will depend largely on the objectives of the modelling exercise. Certainly, although we exploit data-based mechanistic modelling whenever the availability of data allow for this, we also make good use of large simulation models (although normally considered within a stochastic setting and utilizing stochastic simulation: e.g. Parkinson and Young 1998).

Finally, there is one paradox raised by the comparison of our results with those of Wigley that we will leave the reader to contemplate. If the results shown in Wigley's Figure 21.1, which presents the simulated atmospheric $\mathrm{CO}_{2}$ generated by a number of deterministic and rather heavily parameterized simulation models, are compared with those obtained above (Figure 6), then it is clear that, despite the relative complexity of the deterministic models, the simple DBM models (33) or (34) explain the observational data considerably better. Moreover this DBM model, although simple, cannot be dismissed as an exercise in curve fitting. It is not a 'black-box' representation: as we have outlined here and shown more comprehensively in Young and Jarvis (2002), its parameters, as estimated or derived, are physically meaningful; and the physical deductions drawn from these parameters are all reasonable (albeit controversial) from a scientific standpoint. But we must emphasize that the information content of the available data is not high and so these deductions must be judged with due circumspection, as must those derived from the much more complex deterministic climate models.

\section{Conclusions}

This paper provides an introduction to time-domain methods for identifying linear and nonlinear, continuous-time models of stochastic systems from discrete-time sampled data and illustrates the practical utility of these methods for the data-based mechanistic modelling of environmental systems. The main advantage of these methods, over the alternative and better known discrete-time methods, is that they provide differential equation models whose parameters can be interpreted immediately in physically meaningful terms, as our examples have shown. Consequently, they are 
of direct use to environmental scientists and engineers who most often derive models in differential equation terms based on natural (e.g. conservation) laws and who are much less familiar with discrete-time models. Moreover, the continuous-time methods can be adapted easily to handle the case of irregularly sampled data or non-integer time delays that are often encountered in the modelling of real systems. They are also much superior when applied to rapidly sampled data, where discrete-time methods often perform poorly because the eigenvalues lie close to the unit circle in the complex domain, so that the model parameters can be quite poorly defined in statistical terms, leading to estimation problems.

\section{References}

Akaike, H. (1974). A new look at statistical model identification. IEEE Transactions on Automatic Control 19, 716-723.

Aström, K. J. (1969). On the choice of sampling rates in parametric identification of time series. Information Sci. 1(1), 273-278.

Bastogne, T., H. Garnier and P. Sibille (2001). A PMF-based subspace method for continuous-time model identification. Application to a multivariable winding process. International Journal of Control 74(2), 118-132.

Beer, T. and P. C. Young (1983). Longitudinal dispersion in natural streams. Am. Soc. of Civil Eng., Jnl. Env. Eng. 109, 1049-1067.

Belforte, G., F. Dabbene and P. Gay (2005). LPV approximation of distributed parameter systems in environmental modelling. Environmental Modelling \&S Software 20, 1063-1070.

Box, G. E. P. and G. M. Jenkins (1970). Time Series analysis Forecasting and Control. Holden-Day: San Francisco.

Carvalho, Jonas C., Ézio R. Nichimura, Marco Túllio M.B. de Vilhena, Davidson M. Moreira and Gervásio A. Degrazia (2005). An iterative Langevin solution for contaminant dispersion simulation using the GramCharlier PDF. Environmental Modelling \&5 Software 20, 285-289.

Dai, A. and I. Y. Fung (1993). Can climate variability contribute to the missing $\mathrm{CO}_{2}$ sink?. Global Biogeochem. Cycles 7, 599-609. 
Fan, H., T. Söderström, M. Mossberg, B. Carlsson and Y. Zou (1999). Estimation of continuoustime AR process parameters from discrete-time data. IEEE Transactions on Signal Processing 47, $1232-1244$.

Farquhar, G. D., M. J. R. Fasham, M. L. Goulden, M. Heimann, V. J. Jaramillo, H. S. Khesgi, C. Le Quere, R. J. Scholes and D. W. R. Wallace (2001). The carbon cycle and atmospheric carbon dioxide. In: Climate Change 2001: The Scientific Basis, (contribution of working group I to the third assessment report of the Intergovernmental Panel on Climate Change) (J. T. Houghton et al., Eds.). Cambridge University Press: Cambridge (U.K.). pp. 217-222.

Garnier, H. and M. Mensler (2000). The CONTSID toolbox: a Matlab toolbox for CONtinuousTime System IDentification. In: Proceedings, 12th IFAC Symposium on System Identification (SYSID'2000). Santa Barbara (USA).

Garnier, H., M. Gilson and W.X. Zheng (2000). A bias-eliminated least-squares method for continuous-time model identification of closed-loop systems. International Journal of Control 73(1), 38-48.

Garnier, H., M. Mensler and A. Richard (2003). Continuous-time model identification from sampled data. Implementation issues and performance evaluation. International Journal of Control 76(13), 1337-1357.

Garnier, H., P. Sibille and A. Richard (1995). Continuous-time canonical state-space model identification via Poisson moment functionals. In: Proceedings, 34th IEEE Conference on Decision and Control (CDC'95), Vol. 2. New Orleans (USA).

Garnier, H., P. Sibille and T. Bastogne (1997). A bias-free least-squares parameter estimator for continuous-time state-space models. In: Proceddings, 36th IEEE Conference on Decision and Control (CDC'97), Vol. 2. San Diego, California (USA). pp. 1860-1865.

Garnier, H., P. Sibille and T. Spott (1994). Influence of the initial covariance matrix on recursive LS estimation of continuous models via generalised Poisson moment functionals. In: Proceedings, 10th IFAC Symposium on System Identification (SYSID'94). Copenhagen (Denmark).

Granger, C. W. J. and T. Teräsvirta (1993). Modelling Nonlinear Economic Relationships. Oxford University Press. Oxford. 
Jakeman, A. J. and P. C. Young (1979). Refined instrumental variable methods of time-series analysis: Part II, multivariable systems. International Journal of Control 29, 621-644.

Jakeman, A. J., I. G. Littlewood and P. G. Whitehead (1990). Computation of the instantaneous unit hydrograph and identifiable component flows with application to two small upland catchments. Journal of Hydrology 117, 275-300.

Jemni, A. and J. C. Trigeassou (1996). A comparative study of the deterministic accuracy of two identification methods for continuous systems. In: Proceedings, IEEE SMC CESA'96 IMACS Symp. on Modelling, Analysis and Simulation, Vol. 1. Lille (France). pp. 488-493.

Johansson, R. (1994). Identification of continuous-time models. IEEE Transactions on Signal Processing 42(4), 887-896.

Kristensen, N. R., H. Madsen and S. B. Jorgensen (2004). Parameter estimation in stochastic grey-box models. Automatica 40(2), 225-237.

Larsson, E. (2004). Identification of stochastic continuous-time systems. Algorithms, irregular sampling and Cramer-Rao bounds. PhD thesis. Uppsala University, Sweden.

Ljung, L. (2003). Initialisation aspects for subspace and output-error identification methods. In: Proceedings, European Control Conference (ECC'2003). Cambridge (U.K.).

Mendel, J. M. (1969). A priori and a posteriori identification of time varying parameters. In: Proceedings, 2nd IEEE Conference on System Sciences. Hawaii (USA).

Nielsen, J.N., H. Madsen and P.C. Young (2000). Parameter estimation in stochastic differential equations: an overview. Annual Reviews in Control 24, 83-94.

Ordieres, J. B., E. P. Vergara, R. S. Capuz and R. E. Salazar (2005). Neural network prediction model for fine particulate matter on the US Mexico border in El Paso (Texas) and Ciudad Juárez (Chihuahua). Environmental Modelling \& Software 20, 547-559.

Parkinson, S. and P. C. Young (1998). Uncertainty and sensitivity in global carbon cycle modelling. Climate Research 9, 157-174.

Pearson, A. E., Y. Shen and V. Klein (1994). Application of Fourier modulating function to parameter estimation of a multivariable linear differential system. In: Proceedings, 10th IFAC Symposium on System Identification (SYSID'94). Copenhagen (Denmark). pp. 49-54. 
Pham, D.T. (2000). Estimation of continuous-time autoregressive model from finely sampled data. IEEE Transactions on Signal Processing 48, 2576-2584.

Pintelon, R., J. Schoukens and Y. Rolain (2000). Box-Jenkins continuous-time modeling. Automatica 36, 983-991.

Previdi, F. and M. Lovera (2004). Identification on non-linear parametrically varying models using separable least squares. International Journal of Control 77, 1382-1392.

Price, L., P. C. Young, D. Berckmans, K. Janssens and J. Taylor (1999). Data-based mechanistic modelling and control of mass and energy transfer in agricultural buildings. Annual Reviews in Control 23, 71-82.

Priestley, M. B. (1988). Nonlinear and Nonstationary Time Series Analysis. Academic Press. London.

Sagara, S. and Z. Y. Zhao (1990). Numerical integration approach to on-line identification of continuous-time systems. Automatica 26, 63-74.

Saha, D. C. and G. P. Rao (1983). Identification of continuous dynamical systems - The Poisson Moment Functionals (PMF) approach. Springer-Verlag. Berlin.

Saha, D.C. and G.P. Rao (1980). Identification of lumped linear systems in the presence of unknown initial conditions via Poisson moment functionals. International Journal of Control 31, 637644.

Schimel, D., D. Alves, I. Enting, M. Heimann, F. Joos, D. Raynaud and T. M. L. Wigley (1996). $\mathrm{CO}_{2}$ and the carbon cycle. In: Climate Change 1995: The Science of Climate Change, (contribution of working group I to the second assessment report of the Intergovernmental Panel on Climate Change) (J. T. Houghton et al., Eds.). Cambridge University Press: Cambridge (U.K.). pp. 65-86.

Shackley, S., P. C. Young, S. Parkinson and B. Wynne (1998). Uncertainty, complexity and concepts of good science in climate change modelling: are GCMs the best tools?. Climate Change 38, 159-205.

Shinbrot, M. (1957). On the analysis of linear and non linear systems. ASME Trans. 79, 547-552. 
Sinha, N. K. and Rao, G. P., Eds.) (1991). Identification of continuous-time systems. Methodology and computer implementation. Kluwer Academic Publishers. Dordrecht.

Söderström, T. and M. Mossberg (2000). Performance evaluation of methods for identifying continuous-time autoregressive processes. Automatica 36, 53-59.

Söderström, T., H. Fan, B. Carlsson and S. Bigi (1997). Least squares parameter estimation of continuous-time ARX models from discrete-time data. IEEE Transactions on Automatic Control 42(5), 659-673.

Taylor, C. J., A. Chotai and P. C. Young (2000). State space control system design based on nonminimal state variable feedback: further generalization and unification results. International Journal of Control 73, 1329-1345.

Tuan, P. D. (1977). Estimation of parameters of continuous-time Gaussian stationary process with rational spectral density. Biometrica 64, 385-399.

Unbehauen, H. and G. P. Rao (1987). Identification of Continuous Systems. Systems and Control Series. North-Holland. Amsterdam.

Unbehauen, H. and G. P. Rao (1990). Continuous-time approaches to system identification - a survey. Automatica 26(1), 23-35.

Unbehauen, H. and G. P. Rao (1998). A review of identification in continuous-time systems. Annual Reviews in Control 22, 145-171.

Wallis, S. G., P. C. Young and K. J. Beven (1989). Experimental investigation of the aggregated dead zone model for longitudinal solute transport in stream channels. Proc. Inst. of Civil Engrs, Part 2 87, 1-22.

Wang, L. and P. Gawthrop (2001). On the estimation of continuous-time transfer functions. International Journal of Control 74(9), 889-904.

Wigley, T. (2000). Stabilization of $\mathrm{CO}_{2}$ concentration levels. In: The Carbon Cycle (T. Wigley, Ed.). Cambridge University Press: Cambridge (U.K.). pp. 259-276.

Young, P. C. (1964). In flight dynamic checkout - a discussion. IEEE Transactions on Aerospace 2, 1106-1111. 
Young, P. C. (1965a). The determination of the parameters of a dynamic process. Radio and Electronic Eng. (Jnl. Of IERE) 29, 345-361.

Young, P. C. (1965b). Process parameter estimation and self adaptive control. In: Theory of Self Adaptive Control Systems (P. H. Hammond, Ed.). Plenum Press: New York. pp. 118-140.

Young, P. C. (1969a). Applying parameter estimation to dynamic systems: Part I - theory. Control Engineering 16(10), 119-125.

Young, P. C. (1969b). Applying parameter estimation to dynamic systems: Part II - applications. Control Engineering 16(11), 118-124.

Young, P. C. (1970). An instrumental variable method for real-time identification of a noisy process. Automatica 6, 271-287.

Young, P. C. (1978). A general theory of modeling for badly defined dynamic systems. In: Modeling, identification and Control in Environmental Systems (G. C. Vansteenkiste, Ed.). North Holland: Amsterdam. pp. 103-135.

Young, P. C. (1981). Parameter estimation for continuous-time models - a survey. Automatica $17(1), 23-39$.

Young, P. C. (1984). Recursive Estimation and Time-Series Analysis. Springer-Verlag. Berlin.

Young, P. C. (1993). Time variable and state dependent modelling of nonstationary and nonlinear time series. In: Developments in Time Series Analysis (T. Subba Rao, Ed.). Chapman and Hall: London. pp. 374-413.

Young, P. C. (1998). Data-based mechanistic modeling of environmental, ecological, economic and engineering systems. Journal of Environmental Modelling and Software 13, 105-122.

Young, P. C. (1999). Nonstationary time series analysis and forecasting. Progress in Environmental Science 1, 3-48.

Young, P. C. (2000). Stochastic, dynamic modelling and signal processing: time variable and state dependent parameter estimation. In: Nonlinear and Nonstationary Signal Processing (W. J. Fitzgerald, A. Walden, R. Smith and P. C. Young, Eds.). Cambridge University Press: Cambridge. pp. $74-114$. 
Young, P. C. (2001a). Data-based mechanistic modelling of environmental systems. In: Proceedings, IFAC Workshop on Environmental Systems, first plenary session keynote paper. Yokohama (Japan).

Young, P. C. (2001b). The identification and estimation of nonlinear stochastic systems. In: Nonlinear Dynamics and Statistics (A. I. Mees, Ed.). Birkhauser: Boston. pp. 127-166.

Young, P. C. (2002a). Advances in real-time flood forecasting. Philosophical Trans. Royal Society, Physical and Engineering Sciences 360(9), 1433-1450.

Young, P. C. (2002b). Comments on "On the estimation of continuous-time transfer functions". Int. J. Control 75(9), 693-697.

Young, P. C. (2002c). Optimal IV identification and estimation of continuous-time TF models. In: Proceedings, 15th Triennial IFAC World Congress on Automatic Control. Barcelona (Spain).

Young, P. C. (2003). Top-down and data-based mechanistic modelling of rainfall-flow dynamics at the catchment scale. Hydrological Processes 17, 2195-2217.

Young, P. C. (2004). Identification and estimation of continuous-time hydrological models from discrete-time data. In: Hydrology: Science and Practice for the 21st Century, Vol. 1 (B. Webb, Ed.). British Hydrological Society: London. pp. 406-413.

Young, P. C. (2005). Comments on 'identification on non-linear parametrically varying models using separable least squares' by f. previdi and m. lovera: black-box or open box?. International Journal of Control 78, 122-127.

Young, P. C. and A. J. Jakeman (1979). Refined instrumental variable methods of time-series analysis: Part I, SISO systems. International Journal of Control 29, 1-30.

Young, P. C. and A. J. Jakeman (1980). Refined instrumental variable methods of time-series analysis: Part III, extensions. International Journal of Control 31, 741-764.

Young, P. C. and A. J. Jarvis (2002). Data-based mechanistic modelling, the global carbon cycle and global warming. Technical Report TR/177. Centre for Research on Environmental Systems and Statistics. Lancaster University.

Young, P. C. and M. J. Lees (1993). The active mixing volume: a new concept in modelling environmental systems. In: Statistics for the Environment (V. Barnett and K.F. Turkman, Eds.). J. Wiley: Chichester. pp. 3-43. 
Young, P. C., D. J. Pedregal and W. Tych (1999). Dynamic harmonic regression. Jnl. of Forecasting 18, 369-394.

Young, P. C., M. Foster and M. Lees (1993). A direct approach to the identification and estimation of continuous-time systems from discrete-time data based on fixed interval smoothing. In: Proceedings, 12th Triennial IFAC World Congress on Automatic Control, Vol. 10. Sydney (Australia). pp. 27-30.

Young, P. C., P. McKenna and J. Bruun (2002). Identification of nonlinear stochastic systems by state dependent parameter estimation. International Journal of Control 74, 1837-1857.

Young, P. C., S. Parkinson and M. J. Lees (1996). Simplicity out of complexity: Occam's razor revisited. Journal of Applied Statistics 23, 165-210.

Zhan, Xiaoyong (2005). Parallel Fortran-MPI software for numerical inversion of the Laplace transform and its application to oscillatory water levels in groundwater environments. Environmental Modelling \& Software 20, 279-284. 


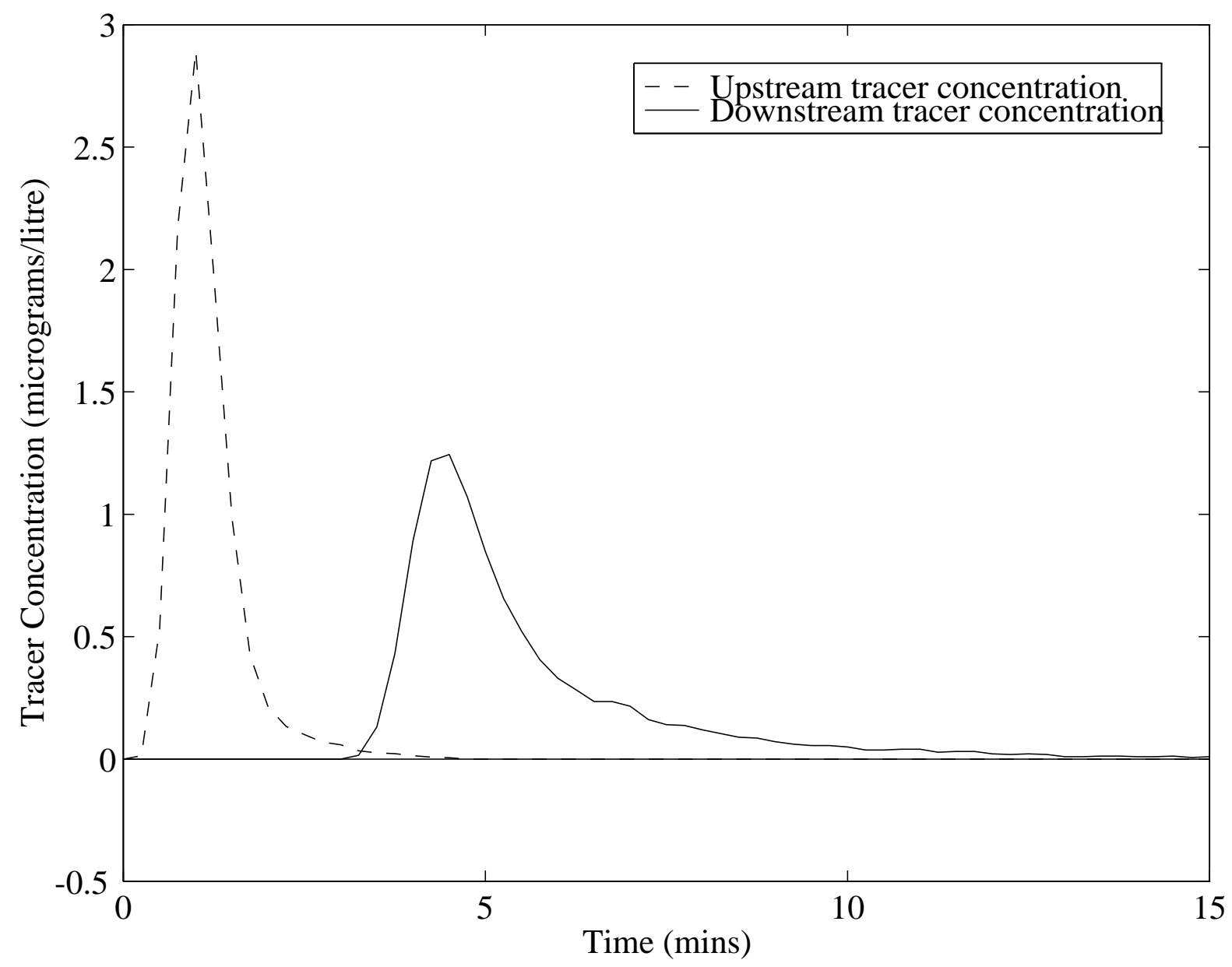

Figure 1: Dye tracer experiment data for the River Conder, North West England: measured input, upstream concentrations (dashed line); measured output, downstream concentrations (full line). 




Figure 2: Comparison of the SRIVC identified ADZ model output $\hat{x}(t)$ (full line) and the measured concentration of tracer $y(t)$ (circular points) at the downstream location. Also shown: inferred quick pathway (dashed) and slow pathway (dash-dot) concentration profiles. 

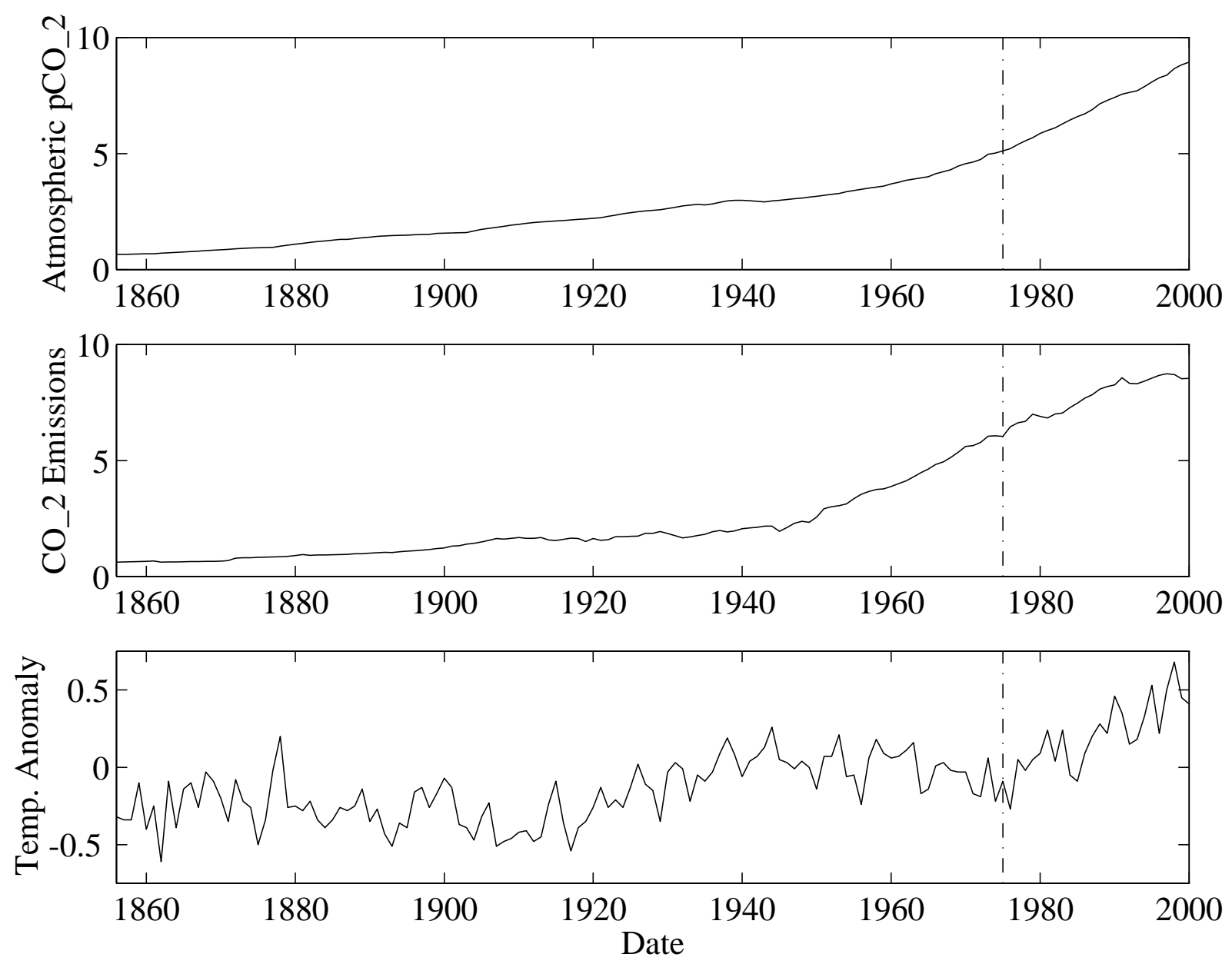

Figure 3: Annual carbon dioxide and temperature anomaly data 1856-2000. Upper panel, perturbations in atmospheric $\mathrm{CO}_{2}$, (measured as partial pressure, $p \mathrm{CO}_{2}$, relative to standard pressure, in pascals, $p a$ ) about an assumed pre-industrial level of $28 \mathrm{pa}$; middle panel, anthropogenic $\mathrm{CO}_{2}$ emissions arising from fossil fuel usage and land use change $\left(G t y^{-1}\right)$; lower panel, Northern Hemisphere average temperature anomaly $\left({ }^{\circ} \mathrm{C}\right)$. The vertical dash-dot line marks the boundary between model estimation (1856-1975) and predictive validation (1976-2000) data (see text). All series are derived from data available at http://cdiac.esd.ornl.gov/. 

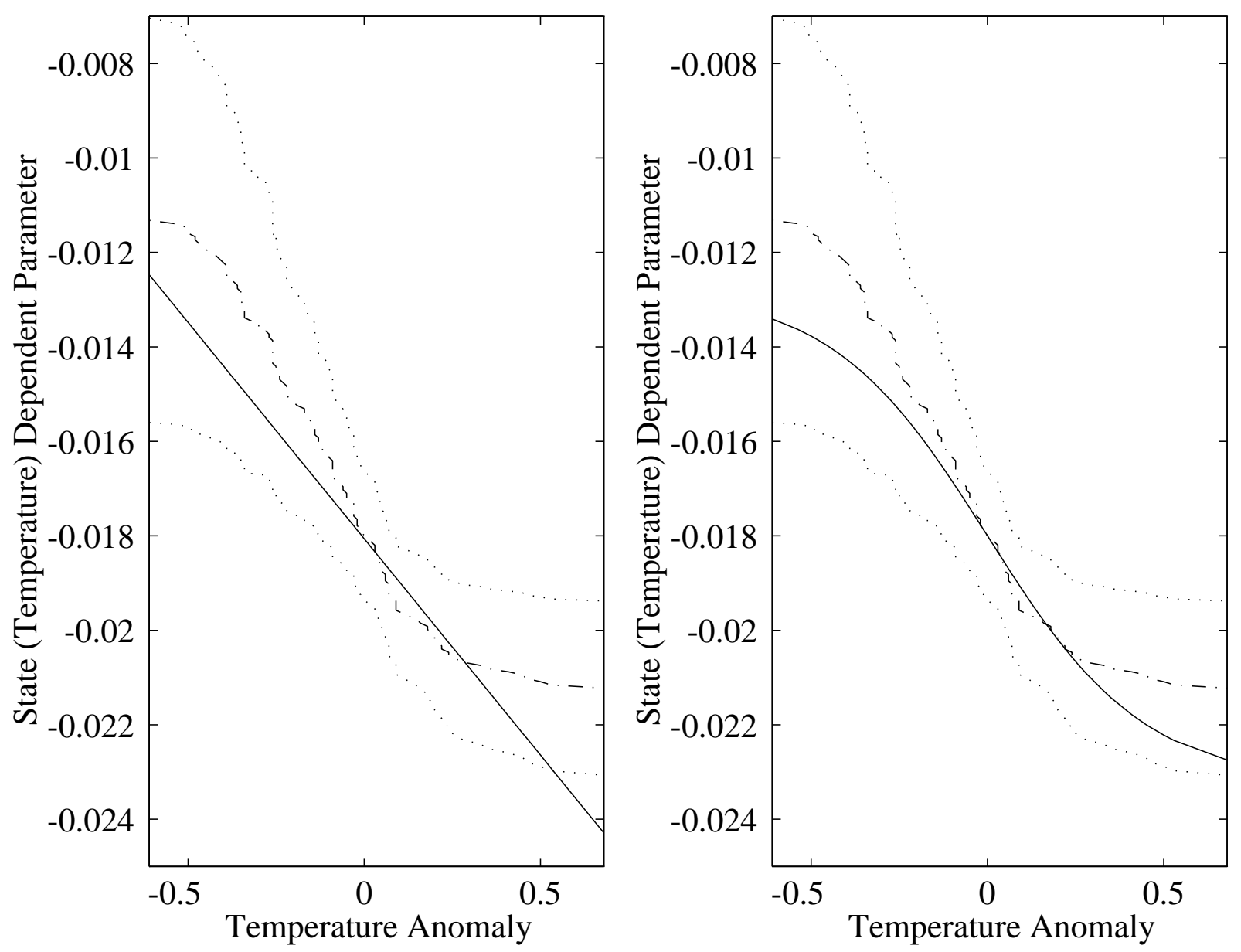

Figure 4: Initial, non-parametric SDP estimate (dash-dot line) and standard error bounds (dots) compared with the parametric model estimates (full lines) from model equations (33) and (34): left panel - linear change with temperature; right panel - sigmoidal change with temperature (cf linear model estimate $\left.\hat{a}_{1}=-0.0167\right)$. 


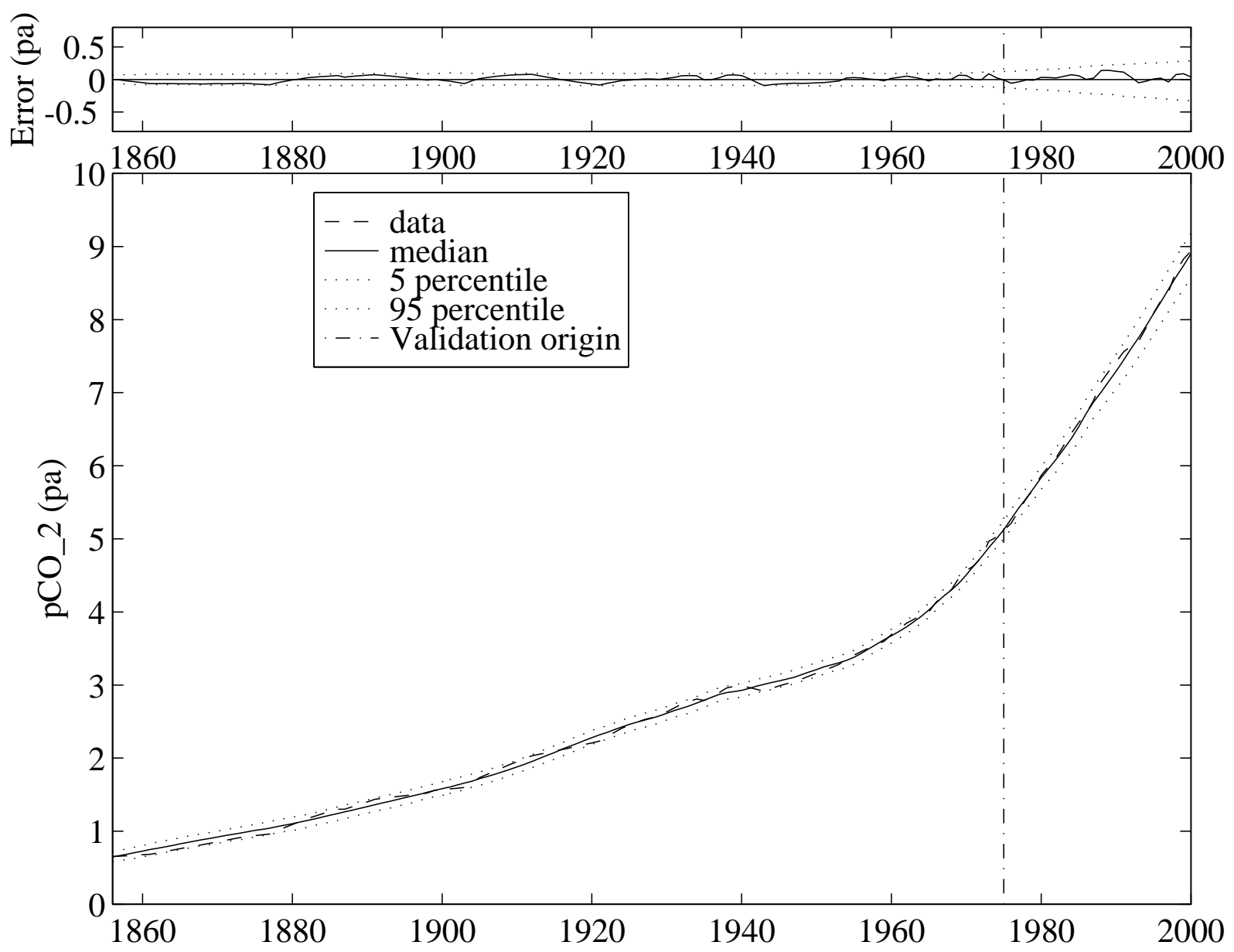

Figure 5: Monte Carlo simulation and predictive validation results: perturbations in atmospheric $\mathrm{pCO}_{2}$ about the assumed pre-industrial level (dashed line); deterministic simulation and MCS median prediction (full line); 5\%-95\% percentile bounds (dotted lines). The error between the simulated and measured $\mathrm{pCO}_{2}$ is shown in the top panel. The vertical dash-dot line marks the boundary between the estimation and validation periods in 1975 . 


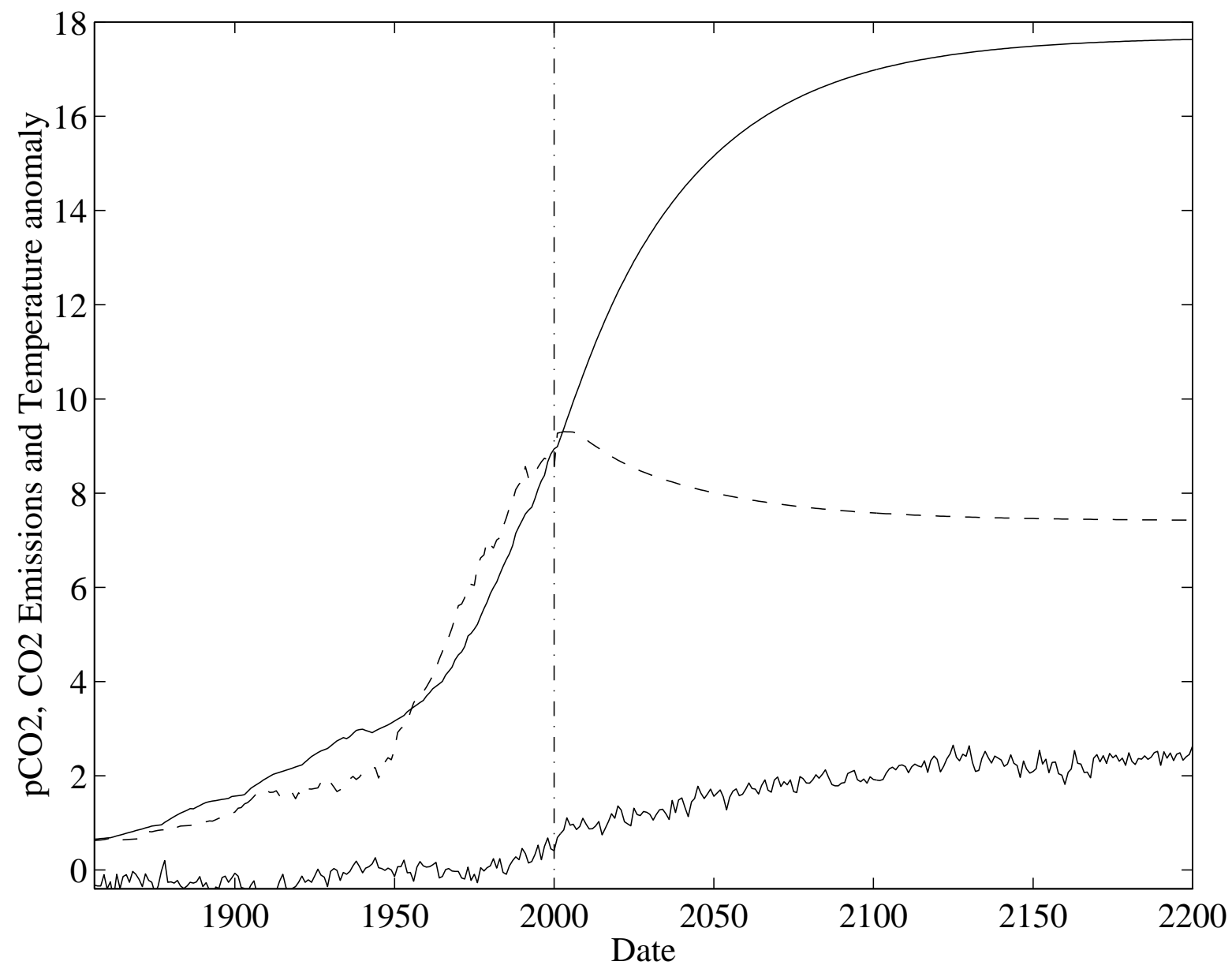

Figure 6: Speculative MCS simulation of the DBM linear model, illustrating how, given this model, a PIP designed automatic control system is able to produce a reasonable $\mathrm{CO}_{2}$ emissions policy (dashed line) that stabilizes 200 year-ahead levels of the simulated atmospheric $p C O_{2}$ perturbations (full line) at a level of 17.6pa (456ppmv) and the simulated temperature anomaly (lower full line) at a mean level of circa $2.6^{\circ} \mathrm{C}$. The vertical dash-dot line marks the initiation of PIP controlled emissions at 2000. 